\title{
MAGNETO-OPTICAL EFFECTS ON THE EMISSION OF SELF-TRAP- PED EXCITON PERTURBED BY Na IMPURITY IN CSI:Na CRYSTAL
}

\author{
J.P. VON DER WEID* and M.A. AEGERTER** \\ Institut de Physique. Université de Neuchâtel, Rue A.-L. Breguet 1, CH-2000 Neuchâtel. \\ Su'itzerland
}

The tunnelling recombination of trapped holes $\left(\mathrm{V}_{\mathrm{K}}\right)$ and electrons $\left(\mathrm{Na}^{\circ}\right)$ pairs in Csl: $\mathrm{Na}$ crystal is studied by optical detection of ESR (DC and transient behavior) and circular dichroism in the $420 \mathrm{~nm}$ emission. A model explains the data and allows to determine the tunnelling probabilities.

\section{Introduction}

The properties of self-trapped excitons (STE) in alkali halides have been extensively studied with several experimental techniques such as optical absorption [1], luminescence [2] and magneto-optical experiments [3], and the model of their structure is now well established, analoguous to a relaxed $\left(V_{K}+e\right)$ center. Less information is available on perturbed self-trapped excitons.

The present work concerns the characteristic blue luminescence observed at $420 \mathrm{~nm}$ in sodium doped cesium iodide either under $X$ irradiation or after X irradiation at low temperature (phosphorescence phenomenon). It was recently shown [4] that this emission arises from the recombination of nearby selftrapped hole ( $\mathrm{V}_{\mathrm{K}}$ centers) and electrons (probably $\mathrm{Na}^{0}$ centers) via a tunnelling process and that the efficiency of the recombination depends on the total electronic spin of the pair, the final emitting state being mainly a singlet state. The present study shows that large temperature-dependent magneto-optical effects are associated with the $420 \mathrm{~nm}$ emission and indicates that a triplet state is also involved in the final emitting states after the tunnelling recombination of the $\mathrm{V}_{\mathrm{K}}-\mathrm{Na}^{0}$ pair; this is confirmed by optical detection of Electron Spin Resonance (ODESR) in the final state.

\section{Experimental procedure}

Single crystals of sodium doped cesium iodide ( 200 to $1000 \mathrm{ppm}$ ) grown in our laboratory, were annealed at $450^{\circ} \mathrm{C}$ then mounted in a $\mathrm{TE}_{101} \mathrm{X}$-band ESR cavity

* Physics Department of the Pontificia Universidade Católica do Rio de Janeiro, C.P. 38071, 20000 Brazil.

** Present address: Instituto de Fisica e Quimicu. Universidade de São Paulo, Sâo Carlos, Brazil. 
placed in a superconducting split coil cryostat (Spectromag SM4, Oxford Instruments). The excitation was obtained from a $150 \mathrm{kV} / 10 \mathrm{~mA}$ tungsten target $\mathrm{X}$ ray tube (Mueller MG 150). The emission spectra were analysed with a JarrellAsh $25 \mathrm{~cm}$ grating monochromator followed by an EMI 9558 QB photomultiplier. In the magnetic circular dichroism (MCD) measurements and optical detection of resonances (ODESR) the $420 \mathrm{~nm}$ luminescence was selected by broad band filters. The MCD signal was obtained with a Morvue photoelastic modulator operating at $50 \mathrm{kHz}$. The microwave field was modulated at frequencies from $3 \mathrm{kHz}$ down to $0.001 \mathrm{~Hz}$ and the transient response of the ODESR signals was analysed with the same technique described previously [5].

\section{Results and discussion}

The luminescence spectrum observed under $\mathrm{X}$ irradiation near liquid helium temperature presents two kinds of emission bands: the two intrinsic STE emission bands ( $290 \mathrm{~nm}$ and $338 \mathrm{~nm}$ ) and the $420 \mathrm{~nm}$ emission, characteristic of the sodium impurity. The relative intensity of these bands depends on the $\mathrm{Na}^{+}$ concentration (partial quenching of the intrinsic bands by the presence of the impurity) and the temperature. The $420 \mathrm{~nm}$ emission arises from the radiative recombination of a self-trapped exciton perturbed by a sodium impurity. The phosphorescence phenomenon [4] observed after the $X$ irradiation appears as a non-radiative tunnelling of $\left(\mathrm{V}_{\mathrm{K}}-\mathrm{Na}^{0}\right)$ pair followed by a radiative electron-hole recombination as a perturbed STE emission.

MCD measurements were made with the magnetic field parallel to either a $\langle 100\rangle,\langle 110\rangle$ or $\langle 111\rangle$ crystalline axis for $1.3 \mathrm{~K}<\mathrm{T}<50 \mathrm{~K}$ and $0<H_{0}<50 \mathrm{KG}$. The results show unambiguously that a triplet state is involved in the final emitting state after the tunnelling recombination of $\mathrm{V}_{\mathrm{K}}-\mathrm{Na}^{0}$ center pairs. However the absolute values of the MCD defined as $\left(I^{-}-I^{+}\right) /\left(I^{-}+I^{+}+I_{\mathrm{DC}}\right)$ are much smaller than those obtained for the intrinsic luminescences in pure CsI at the same temperature [6]. This confirms that a singlet state is also involved in the final state, as previously suggested [4]. The singlet state luminescence affects the MCD ratio since it increases the denominator $\left(I_{\mathrm{DC}}\right)$ and therefore reduces the absolute MCD ratio.

The application of a resonant microwave field can affect the emission properties of this system either in the initial state $\left(\mathrm{V}_{\mathrm{K}}-\mathrm{Na}^{0}\right)$ pair) or in the final one (perturbed STE). The spectrum shown in fig. 1 is independent of the polarization of the emitted light and is assigned to the ODESR of $V_{K}$ centers perturbed by a nearby $\mathrm{Na}^{0}$ defect prior the tunnelling process [7]. The two Gaussian shaped lines correspond to centers which are respectively parallel or perpendicular to the magnetic field. Fig. 2 shows the effect of the microwave field on the $420 \mathrm{~nm}$ MCD signal in the final state and confirms that the MCD signal arises from a triplet state emission whose zero field splitting $\mathrm{D}$ is positive but much 'smaller than the one measured with unperturbed STE in CsI [6].

The separation between the $\sigma^{+}$and $\sigma^{-}$polarized ODESR lines in the $\langle 100\rangle$ spectrum is only $800 \mathrm{G}$. The MCD measured along the magnetic field parallel to a 


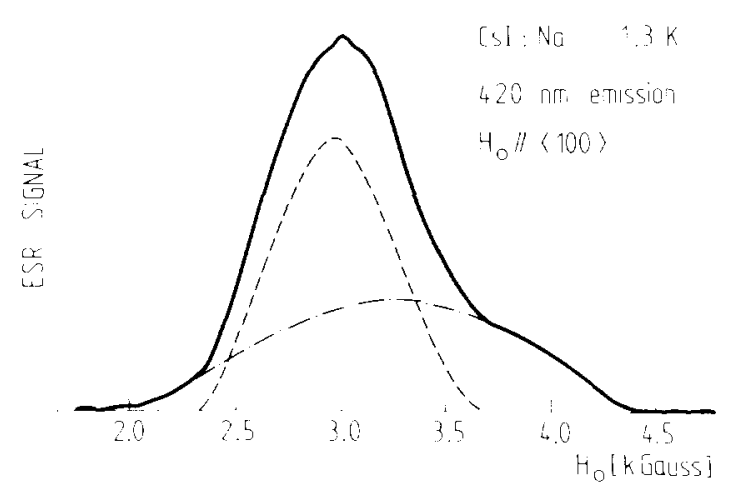

Fig. 1. ESR signal detected optically in the $420 \mathrm{~nm}$ emission of CsI:Nat at $1.3 \mathrm{~K} . \boldsymbol{H}_{10} /(100)$; $\nu=9.1 \mathrm{GHz}$. The spectrum is assigned to perturbed $\mathrm{V}_{\mathrm{K}}$ centers prior the tunnelling.

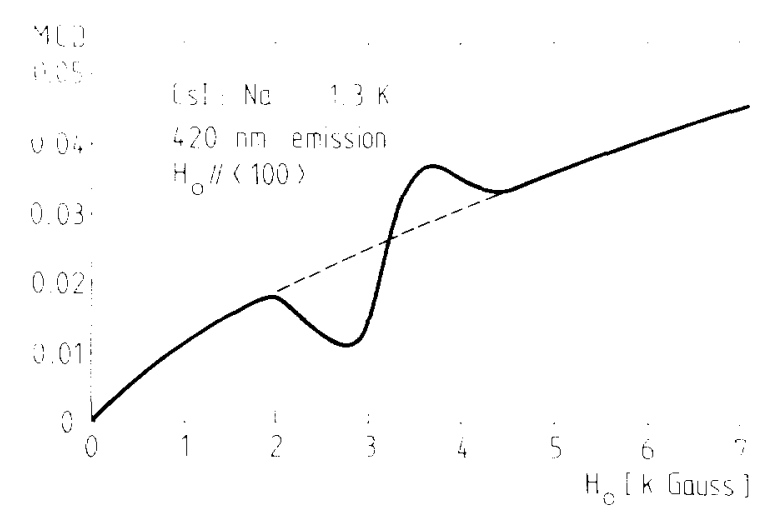

Fig. 2. ESR signal detected optically in the $420 \mathrm{~nm}$ MCD emission of CsI:Nat at $1.3 \mathrm{~K}$. $\left.\boldsymbol{H}_{\mathrm{a}} / /(10)\right)$; $t^{\prime}=9.1 \mathrm{GHz}$. The spectrum is assigned to perturbed self-trapped excitons.

(111) axis gives a polarization which is slightly larger $(\approx 15 \%)$ than along the $\langle 100\rangle$ axis, and the corresponding ODESR also shows the two $\sigma^{+}$and $\sigma$ polarized bands but separated by $980 \mathrm{G}$. These data indicate that the symmetry axis of the perturbed STE is probably away from the $\langle 100\rangle$ axis, and that the zero field splitting is therefore somewhat larger than $980 \mathrm{G}$.

We recently showed [5] that the transient response of the ODESR signal to pulsed microwave excitation gives information about the lifetime of the emitting states as well as about the relaxation times between them. ODESR transient responses measured, prior to the non-radiative tunnelling process, show that the tunnelling probabilities are of the order of $10^{-3} \mathrm{~s}$ while similar measurements in the emitting triplet final state give a lifetime of $6.7 \mathrm{~s}$.

The origin of the ODESR signal in the initial state can be understood with the simple model described in fig. 3. The $\mathrm{V}_{\mathrm{K}}-\mathrm{Na}^{0}$ pairs are not stable: after a tunnelling process. a new emitting state is formed (a perturbed STE) with a 


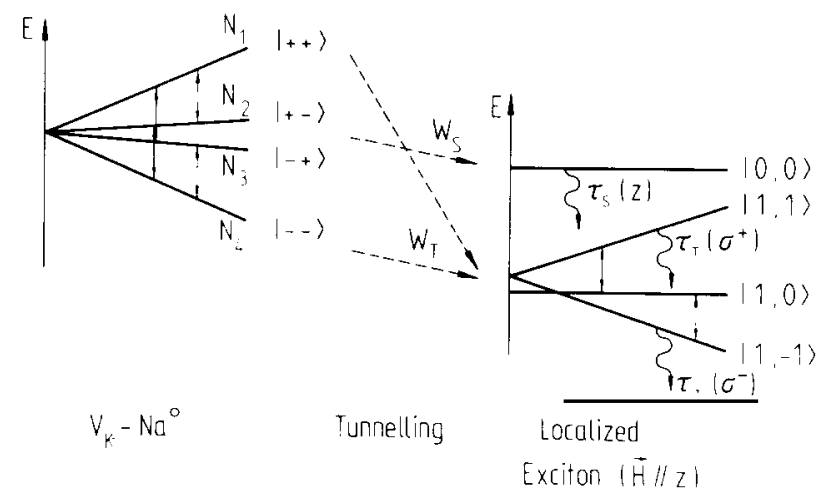

Fig. 3. Schematic diagram of the electronic processes occurring with $\left(\mathrm{V}_{\mathrm{K}}-\mathrm{Na}^{0}\right)$ pairs in CsI: Na. The left side shows the levels of the pairs in a magnetic field before the tunnelling while the right side shows the levels and the radiative transitions of the perturbed self-trapped exciton (only with $\boldsymbol{H} /$ exciton axis) created after the tunnelling process (middle of the figure).

complex radiative decay. This emitting state is formed of a long lived triplet state $\left(\tau_{\mathrm{T}}=6.7 \mathrm{~s}\right)$ located just below a singlet state $\left(\tau_{\mathrm{S}} \cong 10^{-9} \mathrm{~s}\right)$. Let $W_{\mathrm{S}}$ and $W_{\mathrm{T}}$ be the non-radiative tunnelling probabilities to the final singlet and triplet states, $g$ and $g^{\prime}$ the $g$-factors of the $i_{2}^{-}$molecule and $\mathrm{Na}^{0}$ defect, respectively. Neglecting the spin-spin interaction, the energy levels of a pair of defects of $\operatorname{spin} \frac{1}{2}\left(\mathrm{~V}_{K}-\mathrm{Na}^{0}\right.$ pair $)$ prior the tunnelling can be written for a given magnetic field orientation as

$$
E_{1,4}= \pm \frac{1}{2}\left(g+g^{\prime}\right) \beta H, \quad E_{2,3}= \pm \frac{1}{2}\left(g-g^{\prime}\right) \beta H .
$$

If $N_{i}(i=1,4)$ is the population of the four energy levels, the total intensity emitted will be given by $I=\left(N_{1}+N_{4}\right) W_{\mathrm{T}}+\left(N_{2}+N_{3}\right) W_{\mathrm{S}}$.

A microwave field induces spin flips in each component of the pair changing therefore the steady state population $N_{i}^{0}$ of the levels. Thus the intensity of the total emitted light will be changed and its measurement as a function of the field should eventually give the ESR spectra of the $I_{2}^{-}$and $\mathrm{Na}^{0}$ nearby defects. However only ESR lines associated with $I_{2}^{-}$molecules are actually observed. Using the transient response data and the ratio $W_{\mathrm{T}} / W_{\mathrm{S}} \simeq 0.17$ obtained from the magnetic behavior of the phosphorescence intensity [4] we calculate the mean value of the tunnelling probabilities $W_{\mathrm{S}} \approx 1.2 \mathrm{~ms}$ and $W_{\mathrm{T}} \approx 7.0 \mathrm{~ms}$. At the lowest temperature the ODESR signal in the initial state corresponds mainly to the pumping of population from the lowest level $\left(N_{4}\right)$, which present the two spins of the pair in a parallel configuration, into the second one $\left(N_{3}\right)$ with the two spins in antiparallel configuration. The increase in the luminescence intensity is therefore essentially obtained via the singlet emission which is $\sigma$ polarized and do not contribute to the MCD signal. This explains why the ODESR associated with initial state is not observed in the MCD measurements shown in fig. 2 for which only $\pi$ polarized emissions can contribute.

The model gives a good qualitative explanation for the observed data and the estimation of the tunnelling probabilities between $I_{2}{ }_{2}$ and $\mathrm{Na}^{0}$ centers. Furthermore, this is the first report of magneto-optical measurements on perturbed STE 
emissions in alkali-halide; it shows that in CSI the STE perturbed by a sodium impurity presents a long lived triplet state lying slightly below a short lived singlet one and that the perturbation strongly affects the symmetry, lifetimes and zero field splitting of the unperturbed STE.

\section{Acknowledgement}

This work was supported by a grant of the Swiss National Science Foundation.

\section{References}

[1] R.T. Williams and M.N. Kabler, Phys. Rev. B9 (1974) 1897

[2] M.N. Kabler and D.A. Patterson. Phys. Rev. Lett. 19 (1967) 652

[3] W.B. Fowler, M.J. Marrone and M.N. Kabler. Phys. Rev. B8 (1973) 5909

[4] O. Thiébaud. J.J. Pilloud. M.A. Aegerter and C. Jaccard. J. Phys. F 37 (1976) C7. 169.

15] Y. Mori, J.P. von der Weid and M.A. Aegerter. Solid State Commun. (1978) to appear.

[6] L. Falco, J.P. von der Weid and M.A. Aegerter, Int. Conf. on Defects in insulating crystals, Gatlinburg (1977): a more detailed account has been submitted to J. Phys. C (Solid State)

[7] J.P. von der Weid and M.A. Aegerter, Solid State Commun. (1978) to appear 